\title{
A Simple Combination of Univariate Models
}

\author{
Fotios Petropoulos ${ }^{\mathrm{a}, *}$, Ivan Svetunkov ${ }^{\mathrm{b}}$ \\ ${ }^{a}$ School of Management, University of Bath, UK \\ ${ }^{b}$ Centre for Marketing Analytics and Forecasting, Lancaster University Management School, Lancaster, UK
}

\begin{abstract}
This paper describes the approach that we implemented to produce the point forecasts and prediction intervals for the M4-competition submission. The proposed Simple Combination of Univariate Models (SCUM) is the median combination of the point forecasts and prediction intervals of four models, namely Exponential Smoothing, Complex Exponential Smoothing, Automatic Autoregressive Integrated Moving Average and Dynamic Optimised Theta. Our submission performed very well in the M4-competition, being ranked $6^{\text {th }}$ for the point forecasts (with a small difference compared to the $2^{\text {nd }}$ submission) and prediction intervals and $2^{\text {nd }}$ and $3^{\text {rd }}$ for the point forecasts of the weekly and quarterly data respectively.
\end{abstract}

Keywords: M4-competition, ETS, ARIMA, Theta method, Complex exponential smoothing, median combination

\section{The approach}

Our approach is a simple combination of four models. All models were applied using existing implementations in the R statistical software. The following four models were considered (Table 1 summarises the R packages and functions used for each model and frequency):

- Exponential smoothing (ETS, Hyndman et al., 2002), which selects the best model, underlying one of the fifteen exponential smoothing methods based on the minimisation of a pre-specified information criterion. For time series with frequencies lower or equal to 24 (yearly, quarterly, monthly and daily), we used the ets ( ) function of the forecast package (Hyndman et al., 2017); for higher frequencies (weekly and hourly series), we used the es () function of the smooth package (Svetunkov, 2018), which selects the best model out of all the possible 30. The state-space model underlying the function es () differs from the one underlying ets () mainly due to the usage of log normal distribution for multiplicative error models and due to a different transition matrix for the seasonal cases. In addition, es () is able to work on the data with frequencies

\footnotetext{
${ }^{*}$ Correspondence: Fotios Petropoulos, School of Management, University of Bath, Claverton Down, Bath, BA2 7AY, UK.

Email addresses: f.petropoulos@bath.ac.uk (Fotios Petropoulos), i.svetunkov@lancaster.ac.uk (Ivan Svetunkov)
} 
greater than 24. Both functions use by default the corrected Akaike Information Criterion (AICc) for the model selection. However, es () function uses Branch and Bound algorithm, that reduces the pool of models under considerations from 30 to a maximum of 10 . This allows speeding up the selection process without a significant reduction of the accuracy of the selected model.

- Complex exponential smoothing (CES, Svetunkov and Kourentzes, 2018), which sidesteps the ETS taxonomy and produces non-linear trends with a slope depending on the data characteristics. There is a non-seasonal and a seasonal version of this model. The former allows sliding between level and trend without the need for dichotomic selection of components appropriate for time series. The latter captures the type of seasonality (additive or multiplicative) and produces the appropriate forecasts, once again without the need to switch between the two option. The combination of these two models allows capturing complex dynamics in data, sidestepping the ETS taxonomy. So CES is a data-driven model that is supposed to capture well the wide variety of tendencies. We used the auto.ces () function of the smooth package, which makes a selection between two seasonal and one non-seasonal models using AICc (see details in Svetunkov and Kourentzes, 2018).

- Automatic Autoregressive Integrated Moving Average model (ARIMA, Hyndman and Khandakar, 2008), which identifies the best ARIMA model. The automated selection works as follows. First, the appropriate degree of differencing is determined by the Kwiatkowski-Phillips-Schmidt-Shin unit root test. Then, four simple models $(p=q=$ $0, p=q=2$ or $p+q=1$, where $p$ and $q$ refer to the autoregressive and moving average orders of the models respectively) are fitted and the model with the lowest AICc is selected as the temporary best model. A search for a better model involves varying the values of $p$ and $q$ of the temporary best model by \pm 1 ; if a better model is indeed found (a model with lower AICc), then this model becomes the temporary best model. Models with and without the constant are considered when searching for a best model. The search continues until a better model cannot be found. The maximum orders of $p$ and $q$ that are tested are 5. A similar approach is used for the seasonal ARIMA models, but restricting the maximum orders of seasonal AR and MA to 2 . The detailed explanation of the order selection mechanism is given in Hyndman and Khandakar (2008). This mechanism is implemented in the auto.arima() function of the forecast package.

- Dynamic Optimised Theta Model (DOTM, Fiorucci et al., 2016b), which is an extension of the theta method for forecasting (Assimakopoulos and Nikolopoulos, 2000) that achieved the best performance in the M3-competition (Makridakis and Hibon, 2000). The data are first checked for seasonality using an autocorrelation function test of lag that matches the frequency of the data; we opted for a $90 \%$ confidence level, similar to the original implementation of Assimakopoulos and Nikolopoulos (2000). If the data are found to be seasonal, then the seasonality is removed as follows. The seasonal indices are calculated via applying the multiplicative classical decomposition method. Subsequently, the original data are divided with the respective seasonal indices to produce the seasonally adjusted data. The original theta method decomposed 
the seasonally adjusted data into two "theta" lines; the first line is the linear regression line on time and has no curvatures $(\theta=0)$ and the second has double the curvatures of the seasonally adjusted data $(\theta=2)$. According to Assimakopoulos and Nikolopoulos (2000), these two theta lines are able to capture the long- and short-term features of the data. DOTM optimises for each series the $\theta$ value of the theta line that focuses on the short-term curvatures of the seasonally adjusted data. We used the dotm() function of the forecTheta package (Fiorucci et al., 2016a). Note that for the series which are longer than 5000 observations (D2047, D2194 and D4099), DOTM is applied only on the most recent 5000 observations.

Table 1: Forecasting models and the corresponding $\mathrm{R}$ functions.

\begin{tabular}{llll}
\hline Model & Frequency & R package & Function \\
\hline \multirow{2}{*}{ ETS } & $\leq 24$ & forecast 8.2 & ets() \\
& $>24$ & smooth 2.3 .1 & es() \\
CES & All & smooth 2.3 .1 & auto.ces() \\
ARIMA & All & forecast 8.2 & auto.arima() \\
DOTM & All & forecTheta 2.2 & dotm() \\
\hline
\end{tabular}

Table 2 shows the frequencies that we assumed per data set together with the forecasting horizons that were required by the organisers of the M4-competition. Even if some data, such as daily or hourly, could exhibit multiple seasonalities, we have opted for simplicity and modelled each series using a single frequency that is suitable for the relatively short required forecasting horizons.

Table 2: Horizons required and frequencies assumed for each data set.

\begin{tabular}{lcc}
\hline Data set & Horizon & Frequency \\
\hline Yearly & 6 & 1 \\
Quarterly & 8 & 4 \\
Monthly & 18 & 12 \\
Weekly & 13 & 52 \\
Daily & 14 & 7 \\
Hourly & 48 & 168 \\
\hline
\end{tabular}

The median operator was used to combine the outputs of the four models. While more complicated methods for averaging the prediction intervals information from the different models could have been considered, Lichtendahl et al. (2013) claim that a simpler method of averaging the quantiles seems to work quite well. As such, we opt for simplicity and take the median of the upper bounds for all the models and similarly take the median of the lower bounds of the prediction intervals in order to obtain the combined intervals. After averaging the point forecasts and prediction intervals using the median operator, we set any negative values to zero, as the M4-competition data set consists of non-negative values. 
A flowchart of the proposed Simple Combination of Univariate Models (SCUM) approach to produce the point forecasts is depicted in figure 1, where $y$ denotes the in-sample time series data, freq refers to the frequency of this series (for example, 12 for monthly data), $o b s$ is the number of the observations in the in-sample data, $\hat{y}_{m}$ is the point forecast for method $m$ and $\hat{y}$ is the combined point forecast. The upper and lower prediction intervals are produced similarly.

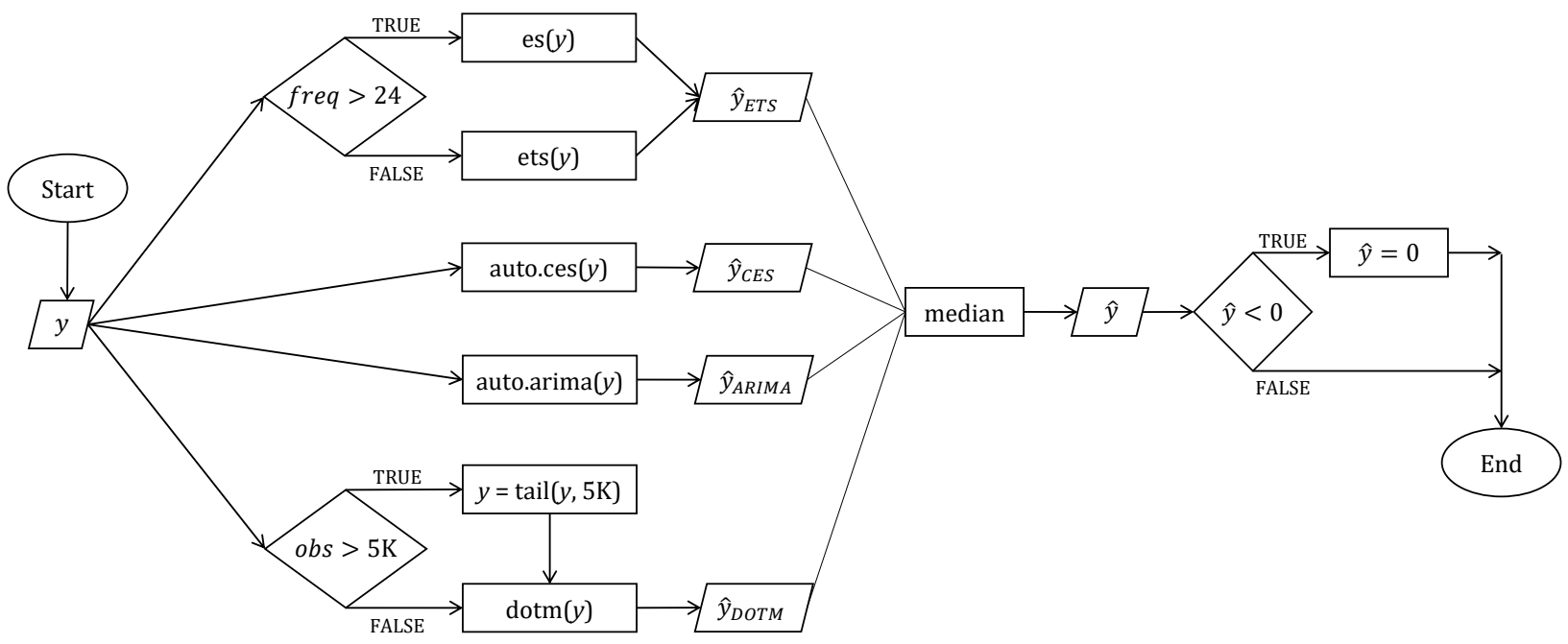

Figure 1: The flowchart of the SCUM approach.

\section{Reproduction information}

We have provided two R scripts that allow the reproduction of the point forecasts and prediction intervals submitted to the M4-competition. These can be found in the GitHub folder of the M4-competition 11 We used R version 3.4 .3 (2017-11-30), forecast version 8.2, smooth version 2.3.1, forecTheta version 2.2. Moreover, a parallel implementation was adopted in our code, where the following packages were used: doSNOW (1.0.16), foreach (1.4.4), snow (0.4-2), iterators (1.0.9).

All forecasts and prediction intervals were produced using the Bath Balena High Performance Computing (HPC) Service at the University of Bath. More specifically, we used Intel Skylake nodes that are dual socket nodes clocked at 2.6GHz. Each Skylake node has $2 \times$ Intel Xeon Gold 6126 CPUs providing 24 cores and 192GB memory (8GB per core) via 6 memory channels per CPU socket.

\section{Computational time}

In our scientific research we focus on forecasting in retail, which motivated a lot of steps in the development of SCUM algorithm. Modern retail settings consist of hundreds

\footnotetext{
${ }^{1}$ https://github.com/M4Competition/M4-methods
} 
of thousands of combinations of stock keeping units and locations. Moreover, as multiple replenishment cycles occur each day, the number of time series forecasts that need to be produced each day by large multinational retailers approaches the 7 digit threshold. With this exponential increase, the time that a method requires to produce forecasts becomes of major importance. In fact, it is argued that any added benefits in accuracy when comparing approach $A$ with approach $B$ (the benchmark) should be balanced against the additional computational time that is needed to compute the forecasts for $A$ compared to $B$ (Nikolopoulos and Petropoulos, 2018). In essence, the excess in computational time can be converted to monetary cost and this can be, ideally, compared against the cost of the error that is saved by using $A$ over $B$.

Table 3 shows the average computational time (in seconds) that is needed to produce forecasts for seasonal monthly simulated series of varying lengths (5, 10, 15 and 20 years of in-sample data; 100 series were simulated in each length) using the SCUM approach. We also present the time that automatic ETS and ARIMA approaches require to produce forecasts on the same data (using the forecast package). The computations were performed on a machine that features Intel Core i7 7500U with 16GB memory and Windows 10 Pro (no parallelisation was applied). We observe that the good performance of SCUM involves only moderate increase in computational time compared to approaches that are widely considered as benchmarks in forecasting research (Petropoulos et al., 2018). In contrast, approaches that involve machine learning elements or selection of models using cross-validation techniques (rolling origin evaluation) will result in a multifold increase of the computational time when retraining of the models is required. An in-depth computational analysis of the SCUM approach compared to other submissions in the M4-competition is provided by Makridakis et al. (2019) in this special issue.

Table 3: Average computational time for producing forecasts (in seconds).

\begin{tabular}{lcccc}
\hline \multirow{2}{*}{ Approach } & \multicolumn{4}{c}{ Observations (in months) } \\
& 60 & 120 & 180 & 240 \\
\hline ETS & 0.542 & 0.632 & 0.732 & 0.812 \\
ARIMA & 0.580 & 2.819 & 1.278 & 1.911 \\
SCUM & 1.884 & 4.489 & 3.313 & 4.285 \\
\hline
\end{tabular}

\section{Why did this approach perform well and how can it be improved?}

In our opinion, the main reasons for the good performance of SCUM are as follows:

- Combination of the models:

The previous competitions (and even this one) showed that the combination of forecasting methods produces more accurate forecasts than the individual ones. This is due to the increase robustness of the final forecasts and the decrease of risks of having the completely incorrect forecasts. While each individual model might fail from time to 
time, their combination tends to be closer to the true value. Table 4 presents the percentage differences in the performance of our approach (SCUM) with each of the four models for each frequency and error measure (symmetric Mean Absolute Percentage Error, sMAPE, and Mean Absolute Scaled Error, MASE) that was used in the M4competition. Positive values suggest that SCUM performs better over the respective approaches. For example, the sMAPE of the ETS for the yearly data is $12.3 \%$ higher than the respective value of SCUM and is calculated as $100 \times \frac{s M A P E_{E T S}-s M A P E_{S C U M}}{s M A P E_{S C U M}}$, where $s M A P E_{E T S}$ and $s M A P E_{S C U M}$ are the sMAPE values for the yearly data for ETS and SCUM respectively. In some cases (notably for yearly data and ETS, CES and ARIMA), the percentage differences are higher than 10\%. It is worth mentioning that CES does not perform well for hourly data, with the respective percentage differences of SCUM over this approach being $124.2 \%$ and $72.8 \%$ for the sMAPE and the MASE respectively. Only in two cases, we observe deterioration in performance compared to a single model; these are DOTM applied on yearly data and ARIMA applied on hourly data when performance is measured by the MASE.

Table 4: Percentage differences in the performance of the combined forecast using the median operator (SCUM) with the four individual models and a combination based on the arithmetic mean.

\begin{tabular}{llccccc}
\hline & Data set & ETS & CES & ARIMA & DOTM & Mean Combination \\
\hline sMAPE & Yearly & $12.3 \%$ & $10.8 \%$ & $11.0 \%$ & $0.1 \%$ & $0.9 \%$ \\
& Quarterly & $5.1 \%$ & $8.0 \%$ & $6.5 \%$ & $3.0 \%$ & $0.7 \%$ \\
& Monthly & $5.2 \%$ & $6.7 \%$ & $4.6 \%$ & $3.6 \%$ & $-0.3 \%$ \\
& Weekly & $5.5 \%$ & $21.4 \%$ & $0.7 \%$ & $14.6 \%$ & $1.5 \%$ \\
& Daily & $4.5 \%$ & $2.1 \%$ & $7.1 \%$ & $2.2 \%$ & $0.8 \%$ \\
& Hourly & $10.5 \%$ & $124.2 \%$ & $4.3 \%$ & $6.2 \%$ & $16.0 \%$ \\
& Overall & $7.1 \%$ & $8.5 \%$ & $6.7 \%$ & $2.6 \%$ & $0.3 \%$ \\
\hline MASE & Yearly & $11.4 \%$ & $13.3 \%$ & $10.2 \%$ & $-0.2 \%$ & $-0.1 \%$ \\
& Quarterly & $3.8 \%$ & $7.2 \%$ & $4.2 \%$ & $6.1 \%$ & $0.2 \%$ \\
& Monthly & $3.8 \%$ & $5.7 \%$ & $2.0 \%$ & $7.2 \%$ & $-0.3 \%$ \\
& Weekly & $7.6 \%$ & $13.9 \%$ & $5.8 \%$ & $15.5 \%$ & $1.2 \%$ \\
& Daily & $8.4 \%$ & $0.4 \%$ & $5.8 \%$ & $1.3 \%$ & $1.8 \%$ \\
& Hourly & $15.4 \%$ & $72.8 \%$ & $-3.9 \%$ & $22.8 \%$ & $5.7 \%$ \\
& Overall & $7.7 \%$ & $9.6 \%$ & $6.5 \%$ & $3.3 \%$ & $0.0 \%$ \\
\hline
\end{tabular}

- Pool of forecasting models:

The forecasting models used in our approach are diverse. ETS is based on time series decomposition, ARIMA captures inter-dependencies in time series, CES focuses on non-linear long-term tendencies in time series, while DOTM employs short-term curvatures and long-term trends. So, each of the model captures something that the others cannot do. As it can be seen in table 4, the ranks of the four models are different for different frequencies of data. At the same time, the combination of such 
different forecasting models leads to the increase in the accuracy in comparison with the individual performance of each of them.

- Median combination:

Instead of using mean, we used median. In essence, the two models that produce the most extreme forecasts are discarded and the final forecast is in between of the two remaining models. Although it might seem that this should not make a difference for such a small pool of models, this allow us to decrease the influence of models that failed in some of time series. For example, if ARIMA produced the forecast with downward trend, while the other models produced the upward one, the median makes sure that the final forecast has the latter. The last column of table 4 shows that the choice of the median operator (as opposed to the mean) indeed resulted in small improvements for the majority of the data frequencies. Table 5 shows the frequencies with which each of the four models contributed in the calculation of the final point forecasts. Note that different models might be used for different forecasting horizons within the same time series. For example, the required forecast horizon for the yearly frequency was six period ahead and the number of yearly series was 23000. This means that we produced 138000 point forecasts combinations for the yearly frequency. Out of these 138000 combinations, 81616 (59.1\%) used ETS, 37891 (27.5\%) used CES, 80077 $(58 \%)$ used ARIMA and 76527 (55.5\%) used DOTM. In a small number of cases, two or more models produced the same forecasts, thus more than two models effectively contributed to the final combined forecast. As a result, the sums per rows in table 5 are slightly higher than $200 \%$ (276111 or $200.08 \%$ for the yearly frequency). Overall, ETS models are more frequently used (56 to almost $65 \%$ for the various subsets), followed by DOTM; CES is the model that is used less often, with a frequency that varies from 27.5 to almost $50 \%$ of the cases.

Table 5: Relative frequencies that each model was used to the calculation of the final point forecasts.

\begin{tabular}{lcccc}
\hline Data set & ETS & CES & ARIMA & DOTM \\
\hline Yearly & $59.1 \%$ & $27.5 \%$ & $58.0 \%$ & $55.5 \%$ \\
Quarterly & $56.3 \%$ & $37.7 \%$ & $52.5 \%$ & $53.7 \%$ \\
Monthly & $57.1 \%$ & $41.9 \%$ & $44.8 \%$ & $56.3 \%$ \\
Weekly & $61.5 \%$ & $49.9 \%$ & $42.2 \%$ & $47.0 \%$ \\
Daily & $63.2 \%$ & $38.8 \%$ & $50.0 \%$ & $48.2 \%$ \\
Hourly & $64.7 \%$ & $35.2 \%$ & $46.2 \%$ & $54.1 \%$ \\
\hline
\end{tabular}

The same principles were used for the interval forecasts, making them robust in comparison with the prediction intervals produced by individual models.

Practices that could have been applied to further enhance the accuracy of SCUM include:

- Optimal selection of in-sample window of data: 
SCUM uses the entire in-sample history of data (in the case of DOTM, the most recent 5000 observations are used). However, one could argue that such long histories might not be relevant when the forecast horizon is relatively short. For example, the longest monthly series consists of 2794 observations when the required forecast horizon is just 18 months ahead. This argument becomes particularly clear in the case of structural changes in the patterns of the data and/or when outlying values have been recorded. In such cases, fitting the models only on a subset of the most recent data that does not include such irregularities might yield more robust forecasts. However, we must underline that this subset should still be long enough to allow for appropriate estimation of the models' parameters and the uncertainty around the forecasts. A strategy for defining an optimal in-sample window for each series could include the application of techniques for identifying structural changes in the historical patterns of the data; in case of structural changes, only the latest data after such changes should be considered for fitting the models. Alternatively, an empirical exercise could shed light on an optimal in-sample window in an aggregate manner (per frequency or category) rather than per individual time series.

- Parameter optimisation that explicitly corresponds to the cost functions:

For all of the models, we used the default settings. This implies the minimisation of Mean Squared Error (MSE) of one-step-ahead forecast. This can be considered as a limitation, because the estimator is not aligned with the error measures used by the organisers of the competition (an average of sMAPE and MASE). If each of the models was estimated via the minimisation of OWA, then there could be potential gains in the accuracy. This also applies to the prediction intervals, which were generated directly from our models. In order to improve the accuracy of the intervals, we could have generated them from the models with the specific estimators driven by the error measure for intervals (Mean Scaled Interval Score).

- Multiple temporal aggregation (MTA):

Many recent studies have shown the positive impact of multiple temporal aggregation on forecasting accuracy (for example, see: Kourentzes et al., 2014; Petropoulos and Kourentzes, 2014; Athanasopoulos et al., 2017). Such improvements are associated with primarily reducing the model uncertainty by expressing the input data in alternative time frequencies. We would expect that an integrated SCUM/MTA approach would further increase forecast accuracy, arguably with an increase in the computational cost.

- Multiple seasonal cycles:

When forecasting series with high frequency, the results could have been possibly improved if models that explicitly consider multiple seasonal cycles were applied. To this direction, the multiple seasonal decomposition function of the forecast package could be used (mslt ()). This would be especially important if the desired horizons were greater than the ones requested by the organisers of the M4-competition. 


\section{Final comment}

As it can be seen from this note, SCUM is a very simple approach that relies on established forecasting models and uses one of the main forecasting principles: combination. The application of SCUM is very straightforward, as the four components of this approach are readily available in the $\mathrm{R}$ statistical software. As it can be seen from the preliminary results of the M4-competition (Makridakis et al., 2018), this simple approach leads to accurate forecasts, placing SCUM on the $6^{\text {th }}$ place in the ladder. A comparison of the SCUM approach with the top-five methods of the M4-competition suggests that the SCUM is probably the simplest forecasting approach among the leaders of the competition, providing a balance between computational complexity and forecasting accuracy.

\section{References}

Assimakopoulos, V., Nikolopoulos, K., 2000. The Theta model: a decomposition approach to forecasting. International journal of forecasting 16 (4), 521-530.

Athanasopoulos, G., Hyndman, R. J., Kourentzes, N., Petropoulos, F., 2017. Forecasting with temporal hierarchies. European Journal of Operational Research 262 (1), 60-74.

Fiorucci, J. A., Louzada, F., Yiqi, B., 2016a. forecTheta: Forecasting Time Series by Theta Models. R package version 2.2 . URL https://cran.r-project.org/package=forecTheta

Fiorucci, J. A., Pellegrini, T. R., Louzada, F., Petropoulos, F., Koehler, A. B., Oct. 2016b. Models for optimising the theta method and their relationship to state space models. International journal of forecasting $32(4), 1151-1161$.

Hyndman, R., Athanasopoulos, G., Bergmeir, C., Caceres, G., Chhay, L., O'Hara-Wild, M., Petropoulos, F., Razbash, S., Wang, E., Yasmeen, F., 2017. forecast: Forecasting functions for time series and linear models. R package version 8.2 .

URL https: //cran.r-project.org/package=forecast

Hyndman, R. J., Khandakar, Y., Jul. 2008. Automatic time series forecasting: The forecast package for R. Journal of statistical software $27(3), 1-22$.

Hyndman, R. J., Koehler, A. B., Snyder, R., Grose, S., Jul. 2002. A state space framework for automatic forecasting using exponential smoothing methods. International journal of forecasting 18 (3), 439-454.

Kourentzes, N., Petropoulos, F., Trapero, J. R., 2014. Improving forecasting by estimating time series structural components across multiple frequencies. International Journal of Forecasting 30 (2), 291-302.

Lichtendahl, K. C., Grushka-Cockayne, Y., Winkler, R. L., Mar. 2013. Is it better to average probabilities or quantiles? Management science 59 (7), 1594-1611.

Makridakis, S., Hibon, M., 2000. The M3-Competition: results, conclusions and implications. International Journal of Forecasting 16 (4), 451-476.

Makridakis, S., Spiliotis, E., Assimakopoulos, V., 2018. The M4 competition: Results, findings, conclusion and way forward. International Journal of Forecasting.

Makridakis, S., Spiliotis, E., Assimakopoulos, V., 2019. The M4 competition: 100,000 time series 61 forecasting methods. International Journal of Forecasting.

Nikolopoulos, K., Petropoulos, F., 2018. Forecasting for big data: Does suboptimality matter? Computers \& Operations Research 98, 322-329.

Petropoulos, F., Kourentzes, N., 2014. Improving forecasting via multiple temporal aggregation. Foresight: The International Journal of Applied Forecasting 34, 12-17.

Petropoulos, F., Wang, X., Disney, S. M., 2018. The inventory performance of forecasting methods: Evidence from the M3 competition data. International Journal of Forecasting.

Svetunkov, I., 2018. smooth: Forecasting Using Smoothing Functions. R package version 2.3.1. URL https://github.com/config-il/smooth 
Svetunkov, I., Kourentzes, N., 2018. Complex exponential smoothing for seasonal time series. Working Paper of Department of Management Science, Lancaster University 2018:1, 1-20.

\section{Acknowledgments}

This project made use of the Balena High Performance Computing (HPC) Service at the University of Bath. 